\title{
Some Remarks and Propositions on Riemann Hypothesis
}

\author{
Jamal Salah \\ Department of Basic Science, College of Health and Applied Sciences, A’Sharqiyah University, Ibra post code 400, Oman
}

Received February 2, 2021; Revised March 17, 2021; Accepted April 9, 2021

\section{Cite This Paper in the following Citation Styles}

(a): [1] Jamal Salah, " Some Remarks and Propositions on Riemann Hypothesis," Mathematics and Statistics, Vol. 9, No. 2, pp. 159 - 165, 2021. DOI: 10.13189/ms.2021.090210.

(b): Jamal Salah (2021). Some Remarks and Propositions on Riemann Hypothesis. Mathematics and Statistics, 9(2), 159 - 165. DOI: 10.13189/ms.2021.090210.

Copyright $\odot 2021$ by authors, all rights reserved. Authors agree that this article remains permanently open access under the terms of the Creative Commons Attribution License 4.0 International License

\begin{abstract}
In 1859, Bernhard Riemann, a German mathematician, published a paper to the Berlin Academy that would change mathematics forever. The mystery of prime numbers was the focus. At the core of the presentation was indeed a concept that had not yet been proven by Riemann, one that to this day baffles mathematicians. The way we do business could have been changed if the Riemann hypothesis holds true, which is because prime numbers are the key element for banking and e-commerce security. It will also have a significant influence, impacting quantum mechanics, chaos theory, and the future of computation, on the cutting edge of science. In this article, we look at some well-known results of Riemann Zeta function in a different light. We explore the proofs of Zeta integral Representation, Analytic continuity and the first functional equation. Initially, we observe omitting a logical undefined term in the integral representation of Zeta function by the means of Gamma function. For that we propound some modifications in order to reasonably justify the location of the non-trivial zeros on the critical line: $s=\frac{1}{2}$ by assuming that $\zeta(s)$ and $\zeta(1-s)$ simultaneously equal zero. Consequently, we conditionally prove Riemann Hypothesis.
\end{abstract}

MSC 2010 Classification: 97I80, 11M41

Keywords Riemann Hypothesis, Analytic Continuity, Functional Equation, Non-trivial Zeros

\section{Introduction}

A mathematical conjecture is first suggested in 1859 and still unproven as of 2021 the Riemann Hypothesis. Often referred to as "the Holy Grail of mathematics," it is arguably the most famous of all unsolved mathematical problems. Despite its miscellaneous applications to many fields of mathematics, it is commonly considered to concern the distribution of prime numbers [1 - 7].

In his publication, Riemann calculated "the number of prime numbers less than a given magnitude a certain meromorphic function on $\mathbb{C}$. But Riemann did not completely clarify his evidence; it took mathematicians decades to check his observations, and we have not yet confirmed any of his estimates on the roots of $\zeta$ to this day [8, 10, 11, 14, and 17].

Even Riemann did not prove that all zeta zeros lie on the line $\operatorname{Re}(s)=1 / 2$. Although there are roughly $10^{13}$ nontrivial zeros lying on the critical line, we cannot take it for granted that Riemann Hypothesis or RH is necessarily true unless a genuine proof or disproof is elaborated $[9,12,18]$.

On the other hand, it is worth noting that there are conflicting opinions not only about the credibility of Riemann Hypothesis, but also about some basic results, for example in $[14,15]$ the author questioned the correctness of the analytic continuity and provided evidences of violating the law of Non-Contradiction (LNC).

Even if assuming the uncertainty or the non sharpness of some results including the analytic continuity of Zeta function, the one may not pass over the recurrence of the non - trivial zeros on the critical line! For that, we may wish not to consider violating the logic but we claim there is a neglecting of some undefined terms or conditions; especially that earlier, Euler had to omit a condition and neglect a logical concept in his computations of $\zeta(-1)=$ 
$-\frac{1}{12}$, which is exactly the same value that Riemann computed late see [13]

Euler first provided the analytic continuity over the critical strip as follows

$$
\begin{gathered}
f(x)=\left(1-2^{1-x}\right) \zeta(x)=\sum_{n=1}^{\infty}(-1)^{n+1} n^{-x} \\
\zeta(x)=\frac{1}{1-2^{1-x}} \sum_{n=1}^{\infty}(-1)^{n+1} n^{-x}
\end{gathered}
$$

Then he considered the following series

$$
1+x+x^{2}+\cdots+x^{n}+\cdots=\frac{1}{1-x}, \quad|x|<1
$$

Differentiating yields

$$
1+2 x+3 x^{2}+\cdots=\frac{1}{(1-x)^{2}}
$$

Euler then omitted the condition that $|x|<1$ and then plugged -1 to derive

$$
\begin{array}{r}
f(-1)=1-2+3-4+\cdots=\frac{1}{4} \rightarrow \zeta(-1) \\
=\frac{1}{1-2^{2}} \times \frac{1}{4}=-\frac{1}{12}
\end{array}
$$

Thus, as we meander down past the window through which we take a voyeuristic look at the Riemann hypothesis, we are well served to consider what the deeper meaning of the Riemann hypothesis really is. We can look at what makes the zeta function and Riemann's conclusions on its behavior so special. For that, we consider Riemann first approach as the main scope of our investigation.

\section{Integral Representation: Riemann's approach}

In this section, we first display the proof of the integral representation of $\zeta(s)$ by the means of its multiplication by $\Gamma(s)$, aiming to detect a gap that allows implementing some modifications:

Lemma 1 [1- 10]

$$
\zeta(s) \Gamma(s)=\int_{0}^{\infty} \frac{t^{s-1}}{\left(e^{t}-1\right)} d t
$$

Proof

$$
\begin{gathered}
\zeta(s)=\sum_{n=1}^{\infty} n^{-s} \quad \operatorname{Re}(s)>1, \\
\Gamma(s)=\int_{0}^{\infty} e^{-\tau} \tau^{s-1} d \tau \quad \operatorname{Re}(s)>1 .
\end{gathered}
$$

Let $\tau=n t \rightarrow d \tau=n d t$ yields.

$$
\Gamma(s)=n^{s} \int_{0}^{\infty} e^{-n t} t^{s-1} d t .
$$

Multiplying $\zeta(s) \Gamma(s)$ Riemann derives

$$
\begin{gathered}
\zeta(s) \Gamma(s)=\sum_{n=1}^{\infty} \int_{0}^{\infty} e^{-n t} t^{s-1} d t=\int_{0}^{\infty}\left(\sum_{n=1}^{\infty} e^{-n t}\right) t^{s-1} d t \\
=\int_{0}^{\infty} \frac{t^{s-1}}{e^{t}-1} d t, \quad \operatorname{Re}(s)>1 .
\end{gathered}
$$

Where the exchange of the integral and the series is justified by the local integrability of the function $t \rightarrow \frac{t^{s-1}}{e^{t}-1}$ at zero; in other words the integral above converges at both end points. As $t \rightarrow 0^{+}, e^{t}-1$ behaves liket, so that the integral behaves like $\int_{0}^{a} t^{s-2} d t$ which converges for $\operatorname{Re}(s)>1$. The Integral converges at the right end point because $e^{t}$ grows faster than any power of $t$.

Remark1 the Integral representation of Zeta function is subject to $t \neq 0$.

Proof

Method 1: Since we computed the sum of the geometric series with radius $r=e^{-t}$ that is we already allowed $n \rightarrow$ $\infty$ :

$$
\sum_{n=1}^{\infty} e^{-n t}=\frac{1}{e^{t}-1}, n \rightarrow \infty
$$

From the substitution: $\tau=n t$ and now $n \rightarrow \infty$, it is clearly that $t \neq 0$. Otherwise we are letting

$$
\tau=\infty \times 0
$$

This is an undefined term.

Method 2:

$$
\sum_{n=1}^{\infty} e^{-n t}= \begin{cases}\infty, & t=0 \\ \frac{1}{e^{t}-1}, & t \neq 0\end{cases}
$$

This implies.

$$
t^{s-1} \sum_{n=1}^{\infty} e^{-n t}= \begin{cases}\infty .0, & t=0 \\ t^{s-1} & t \neq 0 .\end{cases}
$$

Thus, in view of the above, the matter arises is not only to justify exchanging the integral and the sum; but how the one can justify neglecting an undefined term $\tau=\infty \times 0$ when $t=0$ or how to compute the geometric series with radius $r=e^{-t}=1$.

Although the set $\{0\}$ is of measure zero and it does not contribute in the integral of Lemma 1; asserting that $t \neq 0$ plays a crucial role in justifying the location of non-trivial zeros as we will deduce through this approach.

For that, what if we slightly modify the integral representation as follows: 
$\zeta(s) \Gamma(s)=\int_{0}^{\infty} \frac{t^{s-1}}{e^{t}-1} d t, \quad \operatorname{Re}(s)>1$ and $t \neq 0$

Similarly, we plug $1-s$ in (1.1)

$\zeta(1-s) \Gamma(1-s)=\int_{0}^{\infty} \frac{t^{-s}}{e^{t}-1} d t, \operatorname{Re}(s)<0, \quad t \neq 0$

Now, if we make use of (1.1) and (1.2) we can readily deduce an elementary result as follows:

Proposition 1 if $\zeta(s)=\zeta(1-s)=0$ then $\operatorname{Re}(s)=\frac{1}{2}$ Proof

$$
\begin{gathered}
\zeta(s) \Gamma(s)+\zeta(1-s) \Gamma(1-s) \\
=\int_{0}^{\infty} \frac{t^{s-1}+t^{-s}}{e^{t}-1} d t, \quad t \neq 0 \\
\int_{0}^{\infty} \frac{t^{s-1}+t^{-s}}{e^{t}-1} d t=0, t \neq 0
\end{gathered}
$$

One way (Uniqueness not verified) that the integral above vanishes is when the integrand equals zero

$$
t^{s-1}+t^{-s}=0, t \neq 0 \text {. }
$$

Solving for $s$ :

$$
\begin{aligned}
& s=\frac{1}{2}+i \frac{(2 m+1) \pi}{2 \ln t}, \quad t \neq 0, \\
& \ln t \neq 0 \text { and } m \in \mathbb{Z}
\end{aligned}
$$

This implies Riemann Hypothesis i.e., the non-trivial zeros lie on the critical line $\operatorname{Re}(s)=\frac{1}{2}$

Note: Obviously; the conclusion above essentially depends on the truth that $t \neq 0$ which is justified in Remark 1.

But the additional condition $\ln t \neq 0 \rightarrow t \neq$ 1 weakens the result, since there are no rational reasons for not letting $t=1$

\section{Analytic Continuity “Riemann's Approach”}

To expand our previous conclusion, it is worth

$$
\begin{gathered}
\oint_{C} \frac{(-t)^{s-1}}{e^{t}-1} d t=\int_{\rho_{1}} \frac{(-t)^{s-1}}{e^{t}-1} d t+\int_{\rho} \frac{(-t)^{s-1}}{e^{t}-1} d t+\int_{\rho_{2}} \frac{(-t)^{s-1}}{e^{t}-1} d t \\
=\int_{R}^{\epsilon} \frac{\left(t e^{-\pi i}\right)^{s-1}}{e^{t}-1} d t+i \epsilon \int_{0}^{2 \pi} \frac{\left(\epsilon e^{-\pi i} e^{i \theta}\right)^{s-1}}{e^{\epsilon e^{i \theta}}-1} e^{i \theta} d \theta+e^{2 \pi i} \int_{\epsilon}^{R} \frac{\left(t e^{-\pi i} e^{2 \pi i}\right)^{s-1}}{e^{t e^{2 \pi i}}-1} d t \\
=-e_{\epsilon}^{-\pi i(s-1)} \frac{t^{s-1}}{e^{t}-1} d t+i \epsilon \int_{0}^{2 \pi} \frac{\left(\epsilon e^{-\pi i} e^{i \theta}\right)^{s-1}}{e^{\epsilon e^{i \theta}}-1} e^{i \theta} d \theta+e^{(2 \pi i-\pi i)(s-1)} \int_{\epsilon}^{R} \frac{t^{s-1}}{e^{t}-1} d t \\
=\left(e^{\pi i(s-1)}-e^{-\pi i(s-1)}\right) \int_{\epsilon}^{R} \frac{t^{s-1}}{e^{t}-1} d t+i \epsilon \int_{0}^{2 \pi} \frac{\left(\epsilon e^{-\pi i} e^{i \theta}\right)^{s-1}}{e^{\epsilon e^{i \theta}}-1} e^{i \theta} d \theta
\end{gathered}
$$

proceeding to the next well-known results: the analytic continuity and the first functional equation. We propose reviewing the original proofs (not unique) that can be derived by the means of the Hankel contour then residue theorem.

Lemma 2[1- 11] the Riemann Zeta function is meromorphic everywhere, except at a simple pole $\mathrm{s}=1$

Proof

From Lemma 1

$$
\zeta(s) \Gamma(s)=\int_{0}^{\infty} \frac{t^{s-1}}{e^{t}-1} d t
$$

To extend this formula to $\mathbb{C} \backslash\{1\}$, we integrate $(-t)^{s} /$ $\left(e^{t}-1\right)$ over a Hankel contour (Figure 1): a path from $+\infty$ Inbound along the real line to $\epsilon>$ 0 , counterclockwise around a circle of radius $\epsilon$ at 0 , back to $\epsilon$ on the real line, and outbound back to $+\infty$ along the real line, around the circle, $t$ can be parameterized by $t=\epsilon e^{i \theta}, 0 \leq \theta \leq 2 \pi$ and $\epsilon$ is a small arbitrary positive constant that we will let tend to 0 :

$$
\rho_{1}:+\infty \rightarrow \epsilon
$$

$\rho$ : arounr a circle of radius $\epsilon$

$$
\rho_{2}: \epsilon \rightarrow+\infty
$$

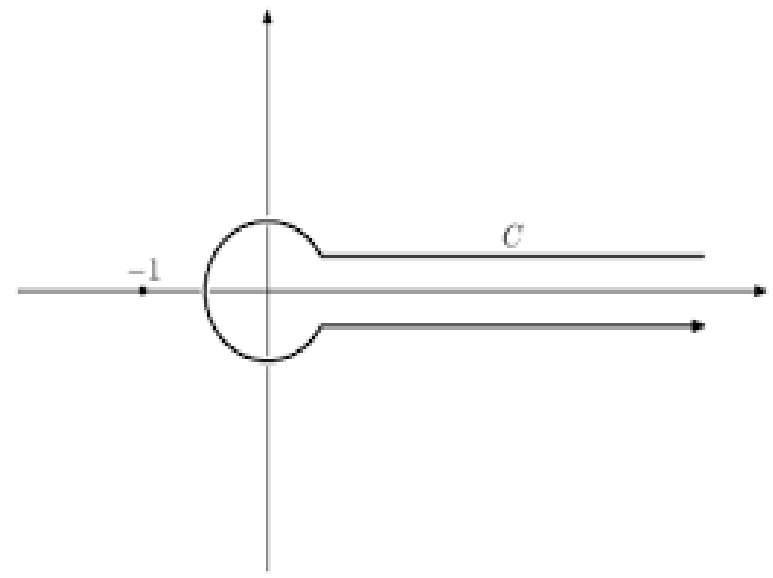

Figure 1. Hankel Contour 


$$
\begin{gathered}
=2 i \sin (\pi s) \int_{\epsilon}^{R} \frac{t^{s-1}}{e^{t}-1} d t+i \epsilon \int_{0}^{2 \pi} \frac{\left(\epsilon e^{-\pi i} e^{i \theta}\right)^{s-1}}{e^{\epsilon e^{i \theta}}-1} e^{i \theta} d \theta \\
=\lim _{\substack{\epsilon \rightarrow 0 \\
R \rightarrow \infty}}\left[2 i \sin (\pi s) \int_{\epsilon}^{R} \frac{t^{s-1}}{e^{t}-1} d t+i \epsilon \int_{0}^{2 \pi} \frac{\left(\epsilon e^{-\pi i} e^{i \theta}\right)^{s-1}}{e^{\epsilon e^{i \theta}}-1} e^{i \theta} d \theta\right], \\
=2 i \sin (\pi s) \int_{0}^{\infty} \frac{t^{s-1}}{e^{t}-1} d t
\end{gathered}
$$

Finally

$$
\oint_{C} \frac{(-t)^{s-1}}{e^{t}-1} d t=2 i \sin (\pi s) \Gamma(s) \zeta(s)
$$

Using Gamma reflection formula

$$
\begin{aligned}
& \Gamma(s) \Gamma(1-s)=\frac{\pi}{\sin (\pi s)} \\
& \zeta(s)=\frac{\Gamma(1-s)}{2 \pi i} \oint_{C} \frac{(-t)^{s-1}}{e^{t}-1} d t
\end{aligned}
$$

Remark 2 By the means of Remark1 and since $t=\epsilon e^{i \theta}$, this implies $|t|=\epsilon \neq 0$. Due to that, we explore the impact of $\epsilon$ all along the integrals above in order to adjust some outputs according to our observation. Now, for sufficiently small nonzero $\epsilon$, the significant change takes place at the integral around the circle that cannot eventually tend to zero. We simply consider the following modifications:

For the integrals along the two paths: $\rho_{1}$ and $\rho_{2}$

$$
2 i \sin (\pi s) \int_{\epsilon}^{R} \frac{t^{s-1}}{e^{t}-1} d t \sim 2 i \sin (\pi s) \int_{0}^{\infty} \frac{t^{s-1}}{e^{t}-1} d t .
$$

The one can consider the left integral as constant times the right one; this will not affect on our purpose, so we simply use the symbol almost equal: instead.

But for the integral around the circle (the path: $\rho$ ) and since we claim that $\epsilon \neq 0$

$$
\int_{\rho} \frac{(-t)^{s-1}}{e^{t}-1} d t=i \epsilon \int_{0}^{2 \pi} \frac{\left(\epsilon e^{-\pi i} e^{i \theta}\right)^{s-1}}{e^{\epsilon e^{i \theta}}-1} e^{i \theta} d \theta=f(s, \epsilon) \epsilon^{s-1}
$$

We omit the term $f(s, \epsilon)$ since it does not play an essential role in the results we are to provide.

Consequently (2.1) and (2.2) can be modified respectively.

$$
\begin{aligned}
& \oint_{C} \frac{(-t)^{s-1}}{e^{t}-1} d t=\epsilon^{s-1}+2 i \sin (\pi s) \Gamma(s) \zeta(s) \\
& \zeta(s)=\frac{\Gamma(1-s)}{2 \pi i} \epsilon^{s-1}+\frac{\Gamma(1-s)}{2 \pi i} \oint_{C} \frac{(-t)^{s-1}}{e^{t}-1} d t
\end{aligned}
$$

\section{The First Functional Equation "Riemann Approach"}

In this section we first complete the consequence of
Lemma 2 as derived by Riemann. Then we establish some modifications in virtue of our previous modifications namely (2.3) and (2.4)

\section{Lemma 3 [7 - 15]}

$$
\zeta(s)=2^{s} \pi^{s-1} \sin \left(\frac{\pi s}{2}\right) \Gamma(1-s) \zeta(1-s)
$$

Proof

Here we consider a modified contour (Figure 2): consisting of two circles centered at the origin and a radius segment along the positive reals. The outer circle has radius $(2 n+1) \pi$ and the inner circle has radius $\epsilon<\pi$. The outer circle is traversed clockwise and the inner one counterclockwise. The radial segment is traversed in both directions. Then employing the residue theorem

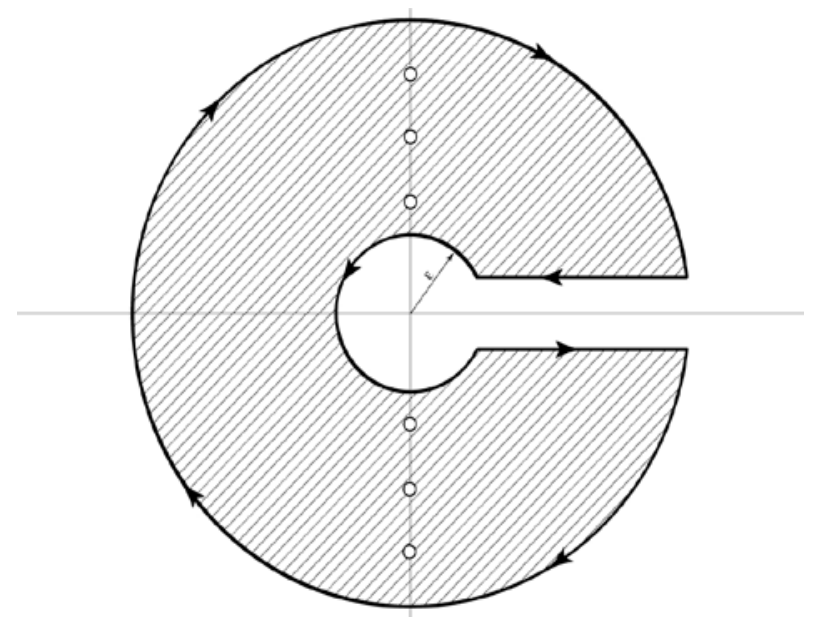

Figure 2. The Modified Contour

$$
\begin{aligned}
\oint_{\gamma} \frac{(-t)^{s-1}}{e^{t}-1} d t= & \lim _{R \rightarrow \infty}\left(\int_{C_{R}} \frac{(-z)^{s-1}}{e^{z}-1} d z\right. \\
& \left.+\int_{D_{R}} \frac{(-z)^{s-1}}{e^{z}-1} d z\right) \\
& =-(2 \pi i) \sum_{n \in Z^{*}} \operatorname{Res}_{z=2 \pi i n} \frac{(-z)^{s-1}}{e^{z}-1}
\end{aligned}
$$

$$
\begin{aligned}
\oint_{\gamma} \frac{(-t)^{s-1}}{e^{t}-1} d t= & -2 \pi i \sum_{n=1}^{\infty}\left(-(-2 \pi i n)^{s-1}\right. \\
& \left.+-(2 \pi i n)^{s-1}\right)
\end{aligned}
$$




$$
\begin{gathered}
=(2 \pi)^{s} \sum_{n=1}^{\infty} n^{s-1} e^{\frac{\pi}{2} i}\left(e^{-i \frac{\pi}{2}(s-1)}+e^{i \frac{\pi}{2}(s-1)}\right) \\
=2^{s} \pi^{s} \zeta(1-s)\left(e^{\frac{\pi}{2} s}-e^{-\frac{\pi}{2} s}\right) \\
=2 i 2^{s} \pi^{s} \zeta(1-s) \sin \left(\frac{\pi}{2} s\right)
\end{gathered}
$$

Plugging in (2.2)

$$
\begin{aligned}
\zeta(s)=\frac{\Gamma(1-s)}{2 \pi i} \oint_{\gamma} \frac{(-t)^{s-1}}{e^{t}-1} d t \\
=\frac{\Gamma(1-s)}{2 \pi i}\left(2 i 2^{s} \pi^{s} \zeta(1\right. \\
\left.-s) \sin \left(\frac{\pi}{2} s\right)\right)
\end{aligned}
$$

That is

$$
\zeta(s)=2^{s} \pi^{s-1} \sin \left(\frac{\pi s}{2}\right) \Gamma(1-s) \zeta(1-s)
$$

From the Functional equation (3.1) the one can readily conclude that $\zeta(-2 n)=0$ these zeros are simply called the trivial Zeta zeros.

\section{Remark 3}

We modify (2.2) by plugging in (2.4)

$$
\begin{gathered}
\zeta(s)=\frac{\Gamma(1-s)}{2 \pi i} \epsilon^{s-1}+\frac{\Gamma(1-s)}{2 \pi i} \oint_{\gamma} \frac{(-t)^{s-1}}{e^{t}-1} d t \\
=\frac{\Gamma(1-s)}{2 \pi i} \epsilon^{s-1} \\
\quad+\frac{\Gamma(1-s)}{2 \pi i}\left(2 i 2^{s} \pi^{s} \zeta(1-s) \sin \left(\frac{\pi}{2} s\right)\right) \\
=\frac{\Gamma(1-s)}{2 \pi i} \epsilon^{s-1} \\
\quad+2^{s} \pi^{s-1} \sin \left(\frac{\pi s}{2}\right) \Gamma(1-s) \zeta(1-s)
\end{gathered}
$$

For simplicity, we omit the coefficient $\frac{\Gamma(1-s)}{2 \pi i}$ and we replace (3.1) by

$$
\zeta(s)=\epsilon^{s-1}+2^{s} \pi^{s-1} \sin \left(\frac{\pi s}{2}\right) \Gamma(1-s) \zeta(1-s)
$$

Similarly

$$
\zeta(1-s)=\epsilon^{-s}+2^{1-s} \pi^{-s} \sin \left(\frac{\pi(1-s)}{2}\right) \Gamma(s) \zeta(s)
$$

Next, we express the assertions (3.2) and (3.3) in terms of $\epsilon^{s-1}$ and $\epsilon^{-s}$ respectively, for shorthand we let.

$$
A(s)=2^{s} \pi^{s-1} \sin \left(\frac{\pi s}{2}\right) \Gamma(1-s)
$$

So, we write

$$
\begin{gathered}
\zeta(s)-A(s) \zeta(1-s)=\epsilon^{s-1} \\
\zeta(1-s)-A(1-s) \zeta(s)=\epsilon^{-s}
\end{gathered}
$$

We add the last two expressions.

$$
\begin{gathered}
\zeta(s)-A(s) \zeta(1-s)+\zeta(1-s)-A(1-s) \zeta(s) \\
=\epsilon^{s-1}+\epsilon^{-s}
\end{gathered}
$$

Proposition 2 given that $\zeta(s)=\zeta(1-s)=0$ then $\operatorname{Re}(s)=\frac{1}{2}$

Proof

$$
\begin{gathered}
\zeta(s)-A(s) \zeta(1-s)+\zeta(1-s)-A(1-s) \zeta(s) \\
=\epsilon^{s-1}+\epsilon^{-s}
\end{gathered}
$$

The only and unique way that

$$
\begin{gathered}
\epsilon^{s-1}+\epsilon^{-s}=0 \\
s=\frac{1}{2}+i \frac{(2 m+1) \pi}{2 \ln \epsilon}, \quad \epsilon \neq 0, \\
\ln \epsilon \neq 0 \text { and } m \in \mathbb{Z}
\end{gathered}
$$

This is also equivalent to RH.

Note: In the conclusion above and since $\epsilon$ is an arbitrary constant then the two conditions $\epsilon \neq 0, \ln \epsilon \neq 0$ are both justified $\epsilon \neq 0$ by Remark 3 and $\ln \epsilon \neq 0 \rightarrow$ $\epsilon \neq 1$ by assumption that $\epsilon$ is an arbitrary constant, so we can simply let $0<\epsilon<1$.

Certainly, we can compute the non-trivial Zeta zeros from the expression.

$$
\begin{aligned}
& s=\frac{1}{2}+i \frac{(2 m+1) \pi}{2 \ln \epsilon}, \quad \epsilon \neq 0, \\
& \ln \epsilon \neq 0 \text { and } m \in \mathbb{Z} .
\end{aligned}
$$

However, the result is weak compared to well-known techniques such that the Euler-Maclaurin summation formula, the Riemann Siegel formula and the Odzyklo-Schölange algorithm [1 - 9].

In Lemma 3 Riemann considered computing the residues at the points of the singularity (the zeros of the denominator: $e^{z}-1$ ) at $z=2 \pi i n$ by allowing $t=0$.

Even though, we have claimed that $t$ cannot be zero, which means that the integral in Lemma 3 is identically zero (in the sense there are no residues), we have proposed modifications on the first functional equation in order to derive some alternative functional equations that describe the structure of the additional terms which are $\epsilon^{s-1}$ and $\epsilon^{-s}$, hence justifying the location of non-trivial zeros at $\operatorname{Re}(s)=\frac{1}{2}$.

\section{An Additional Remark}

For $\tau=n t$ if we omit the condition that $t \neq 0$ we must assert another condition, $n<\infty$ this will provide a sharp Integral Representation. 


$$
\begin{gathered}
\Gamma(s)=n^{s} \int_{0}^{\infty} e^{-n t} t^{s-1} d t, \quad n<\infty, \\
\zeta(s) \Gamma(s)=\sum_{n=1}^{N} \int_{0}^{\infty} e^{-n t} t^{s-1} d t+\sum_{n=N+1}^{\infty} n^{-s}=\int_{0}^{\infty}\left(\sum_{n=1}^{N} e^{-n t}\right) t^{s-1} d t+\sum_{n=N+1}^{\infty} n^{-s} \\
=\int_{0}^{\infty} \frac{t^{s-1}}{e^{t}-1} d t-\int_{0}^{\infty} \frac{e^{-N t} t^{s-1}}{e^{t}-1} d t+\sum_{n=N+1}^{\infty} n^{-s}, \quad \operatorname{Re}(s)>1
\end{gathered}
$$

We did not elaborate further on the modification above, since the purpose was to continue decorticating Riemann Approach in which he already assumed $n \rightarrow \infty$, so we completed the study by asserting that $t \neq 0$.

\section{Conclusion}

In section 1 of this article, we demonstrated a rational reason to impose the condition $t \neq 0$ in the Zeta integral representation. Consequently, we proposed an approach that may shed the light on Riemann Hypothesis. Although, we did not verify the uniqueness nor the condition $t \neq$ 1 of the way the integral in Proposition 1 equal zero, we have shown that the non-trivial zeros lie on the critical line.

We evoked the same concern while investigating the analytic continuity of Zeta function via the Hankel contour, which is $\epsilon$ the radius of the circle, cannot be zero. For that, we proposed keeping a track on the impact of $\epsilon^{s-1}$ in Lemma 2. Consequently, we suggested slight modifications on the functional equations. That allowed providing a unique way for $\epsilon^{s-1}+\epsilon^{-s}=0$ in Proposition 2. In both propositions we have set an initial condition that $\zeta(s)$ and $\zeta(1-s)$ simultaneously equal zero.

Undoubtedly, Riemann derived sufficiently outstanding results in his approach investigating the distribution of prime numbers by the means of complex analysis. Since his purpose was beyond just computing the Zeta zeros, we assume that he may implicitly consider some accurate but not sharp approximations. For that, we may wish to readdress Riemann Hypothesis as follows:

$$
\begin{gathered}
\zeta(s) \Gamma(s) \sim \int_{0}^{\infty} \frac{t^{s-1}}{\left(e^{t}-1\right)} d t \\
\zeta(s) \sim 2^{s} \pi^{s-1} \sin \left(\frac{\pi s}{2}\right) \Gamma(1-s) \zeta(1-s) \\
\zeta(-2 n) \sim 0
\end{gathered}
$$

If there exist s such that $\zeta(s)=\zeta(1-s) \sim 0 \rightarrow s$

$$
=\frac{1}{2}+i y
$$

\section{Conflicts of Interest}

This manuscript has not been submitted to, nor is under review at, another journal or other publishing venues.

\section{REFERENCES}

[1] A. Granville, 8. Riemann's plan for proving the Prime Number Theorem, Andrew Granville's Home Page: Course Notes: Prime Numbers, Université de Montréal. http://www.dms.umontreal.ca/ andrew/Courses/Chapter8. pdf (accessed February 21, 2007).

[2] Bombieri, Enrico (2000), The Riemann Hypothesis - official problem description (PDF), Clay Mathematics Institute,https://www.claymath.org/sites/default/files/offici al_problem_description.pdf (Retrieved 2008-10-25).

[3] Borwein, Peter; Choi, Stephen; Rooney, Brendan; Weirathmueller, Andrea, eds. (2008), The Riemann Hypothesis: (first ed.,) A Resource for the Afficionado and Virtuoso Alike, CMS Books in Mathematics, New York: Springer, 2008, pp 1- 533. Doi: 10.1007/978-0-387-72126-2, ISBN 978-0-387-72125-5.

[4] E. C. Titchmarsh, The theory of the Riemann zeta-function, The Clarendon Press, Oxford University Press, New York, second ed., 1986. , pp 1- 211. Edited and with a preface by D. R. Heath-Brown.

[5] Harold M. Edwards. Riemann Zeta Function. Dover Publications, Dover Ed edition (June 13, 2001), pp 1- 336.

[6] H. Iwaniec Lectures on the Riemann zeta function, vol. 62 of University Lecture Series, American Mathematical Society, Providence, RI, 2014, Volume 53, Number 3, July 2016, Pages 507-512 http://dx.doi.org/10.1090/bull/1525

[7] J. B. Conrey, the Riemann hypothesis, Notices Amer. Math. Soc., March (2003), 341-353.

[8] J. Derbyshire; Prime obsession: Bernhard Riemann and the greatest unsolved problem in mathematics; Joseph Henry Press, Washington D.C., 2003, pp 1- 446 DOI: https://doi.org/10.17226/10532

[9] J. E. Littlewood. The Riemann Hypothesis the Scientist Speculates, New York: Basic books, 1962

[10] Jekel, David. Ithe Riemann Zeta Function. The University of Washington. n.p. Web. Https: ==www: math: Washington: $\mathrm{Edu}=$ morrow=33613=papers=david:pdf (6 June 2013)

[11] Jamal Y. Mohammad Salah, The consequence of the analytic continuity of Zeta function subject to an additional term and a justification of the location of the non-trivial zeros, International Journal of Science and Research (IJSR). 
Volume 9, Issue 3, Pages 1566 - 1569. 2020.

[12] Jamal Y. Mohammad Salah, Two Conditional proofs of Riemann Hypothesis, International Journal of Sciences: Basic and Applied Research (IJSBAR). Volume 49, Issue 1, Pages 74-83. 2020.

[13] Raymond Ayoub, Euler and Zeta function, The American Mathematical Monthly, Volume 81, No. 10 (Dec., 1974), pp. 1067-1086. Published by: Mathematical Association of America Stable URL: http://www.jstor.org/stable/2319041, Accessed: 10-11-2017 17:23 UTC

[14] S. J. Patterson, An introduction to the theory of the Riemann zeta function, vol. 14 of Cambridge Studies in Advanced Mathematics, Cambridge University Press, Cambridge, 1988, pp $1-156$.
[15] Sharon, A. Analytic Continuation of $\zeta(s)$ Violates the Law of Non-Contradiction

(LNC), https://arxiv.org/pdf/1802.08062v7.pdf. 30 July 2019.

[16] Sharon, A. Sharon, A. Applying the law of identity (loi), the law of non-contradiction (lnc), and the law of the excluded middle (lem) to the Dirichlet and Riemann versions of the zeta function, https://arxiv.org/pdf/1802.08062v4.pdf. 28 Jun 2018.

[17] Shigeki Akiyama, Shigeki Egami, and Yoshio Tanigawa. Analytic continuation of multiple zeta-functions and their values at non-positive integers. Acta Arithmetica-Warszawa, 98(2):107-116, 2001

[18] M. du Sautoy, The music of the primes: Searching to solve the greatest mystery in mathematics, Perennial, New York, 2003, pp 1 - 335. 